congenital, genetic, and metabolic disorders in this age group. Future studies should clarify the role of CSE in mesial temporal sclerosis and seizures, and identify specific ethnic, genetic, or socioeconomic factors in etiology and methods of CSE prevention. (RaspallChaure M, Chin RFM, Neville BG, Bedford H, Scott RC. The epidemiology of convulsive status epilepticus in children: a critical review. Epilepsia Sept 2007;48:1652-1663). (Reprints: Dr Rod C Scott, Senior Lecturer in Paediatric Neurosciences, Paediatric Neurologist, Institute of Child Health, The Wolfson Centre, Mecklenburgh Square, London WC1 N2AP, UK).

COMMENT. In a current Dutch study of SE in children with epilepsy, $41(8.3 \%)$ of 494 had one or more episodes of SE at the time of epilepsy diagnosis. Three had febrile SE, followed by unprovoked seizures, and 38 were unprovoked SE. (Stroink H, et al. Epilepsia Sept 2007;48:1708-1715). SE was the first seizure in 32 (78\%). After 5 year follow-up, $31.7 \%$ of patients with $\mathrm{SE}$ at onset had a shorter terminal remission $<1$ year compared to $21.2 \%$ of those without SE. Mortality was not significantly increased for children with SE. Risk factors for SE at onset of epilepsy were remote symptomatic and cryptogenic etiology, and a history of febrile seizures. SE during the course of the epilepsy has a worse prognosis and a high recurrence rate of SE.

\title{
SIMPLE FEBRILE SEIZURES AND TEMPORAL LOBE EPILEPSY ASSOCIATED WITH SCN1A MUTATION
}

The electroclinical features of a large Italian family with 13 living members having simple febrile seizures and SCN1A missense mutation (M145T), some complicated by temporal lobe epilepsy, are reported from University Magna Graecia, Catanzaro, and other centers in Italy. All 13 affected members ( 7 male, mean age $36.6+/-20.4$ years) had febrile seizures up to the age of 6 years. Age at onset of febrile seizures ranged from 5 to 45 months, mean age of $12.8+/-12.9$ months. EEGs were normal in 9 with simple febrile seizures only. Three developed temporal lobe epilepsy, 2 having MRI evidence of mesial sclerosis. (Colosimo E, Gambardella A, Mantegazza M, et al. Electroclinical features of a family with simple febrile seizures and temporal lobe epilepsy associated with SCN1A loss-of-function mutation. Epilepsia Sept 2007;48:1691-1696). (Reprints: Prof Antonio Gambardella, Cattedra ed UO di Neurologia, Universita degli Studi Magna Graecia, Campus Universitario di Germaneto, Viale Europa, 88100 Catanzaro, Italy).E-mail: a.gambardella@isn.cnr.it

COMMENT. SCN1A mutations may cause simple febrile seizures later associated with temporal lobe epilepsy, and separate from the characteristic association with generalized epilepsy with febrile seizures plus (GEFS+).

A frequency of $71 \%$ SCN1A abnormalities are associated with severe myoclonic epilepsy of infancy (SMEI) including borderline SMEI (SMEB), and $11.5 \%$ with GEFS+ probands. (Marini $\mathrm{C}$ et al. Idiopathic epilepsies with seizures precipitated by fever and SCN1A abnormalities. Epilepsia Sept 2005;48:1678-1685). 